\title{
Cathodoluminescence study of laser recrystallized CdTe layers
}

\author{
P. Fernández and J. Piqueras \\ Departamento Física de Materiales, Facultad Físicas, Università Complutense, 28040 Madrid, Spain
}

N. V. Sochinskii and V. Muñoz

Departamento Física Aplicada e Instituto de Ciencia de Materiales de la Universidad de Valencia

(ICMUV), C/Doctor Moliner, 50, 46100 Burjasot, Valencia, Spain

\author{
S. Bernardi \\ Consorzio C.R.E.O., Via Pile 60, I-67100 L'Aquila, Italy
}

(Received 22 July 1997; accepted for publication 25 September 1997)

\begin{abstract}
$\mathrm{CdTe}(100) / \mathrm{GaAs}(100)$ and $\mathrm{CdTe}(111) / \mathrm{CdTe}(111)$ layers grown by metalorganic vapor phase epitaxy (MOVPE) were investigated. The layers were recrystallized to improve their morphology by scanning the surface with a $100 \mu \mathrm{m}$ diameter spot from an $\operatorname{Ar}$ ion laser beam $(\lambda=514.4 \mathrm{~nm})$. Cathodoluminescence spectra from both as-grown and recrystallized CdTe MOVPE layers are used to monitor the effect of the recrystallization procedure. The laser recrystallization results in important changes on the spectral distribution of luminescence. Deep-level bands associated to different defects are shown to be very sensitive to the laser recrystallization procedure. The effect of the different substrates on the defect structure of the layers is also related to the changes observed in the cathodoluminescence spectra. (C) 1997 American Institute of Physics.
\end{abstract}

[S0003-6951(97)01947-5]

Metalorganic vapor phase epitaxy (MOVPE) is a technique commonly used to grow CdTe layers on different lattice mismatched substrates like $\mathrm{Si}, \mathrm{GaAs}$, sapphire, etc. ${ }^{1}$ These layers are used as substrates for subsequent epitaxial growth of $\mathrm{Hg}_{1-x} \mathrm{Cd}_{x}$ Te layers for infrared applications (for recent examples, see Refs. 2-5). It is widely accepted that the properties of CdTe MOVPE layers are strongly dependent on several technological factors, among which the substrate nature is one of the most important. ${ }^{6}$ In particular, previous studies of $\mathrm{CdTe} /$ sapphire and $\mathrm{CdTe} / \mathrm{GaAs}$ layers grown by a low-temperature MOVPE have shown that the as-grown layers have a low quality, rough surface due to strain raised from the large lattice mismatch. ${ }^{6-8}$ To overcome this problem, postgrowth laser recrystallization has been recently proposed as a method to improve the surface morphology of CdTe MOVPE layers. ${ }^{8}$ Nevertheless, it is still an open question how the laser recrystallization procedure affects other properties of the layers besides surface morphology.

Cathodoluminescence (CL) microscopy has been shown to be a very useful tool to characterize CdTe bulk crystals submitted to different technological treatments like thermal annealing, ${ }^{9-11}$ doping, ${ }^{12}$ or epitaxial overgrowth. ${ }^{13}$ The capability to obtain both spectral and spatial resolution allows us to gain quite valuable information about the defect structure of the material. Though bulk crystals have been extensively studied by luminescence techniques, not so much work has been done to characterize CdTe epitaxial layers. In this letter, we report the results on cathodoluminescence studies of asgrown and laser recrystallized $\mathrm{CdTe} / \mathrm{GaAs}$ and $\mathrm{CdTe} / \mathrm{CdTe}$ MOVPE layers and show the effect of the laser recrystallization on the radiative defects contributing to the CL emission of the layers. The influence of the substrate on the behavior of the epilayers under laser irradiation is also reported.

The samples investigated were $\mathrm{CdTe}(100)$ and $\mathrm{CdTe}(111)$ layers grown on $\operatorname{GaAs}(100)$ and $\mathrm{CdTe}(111)$ sub- strates by MOVPE. The layer thicknesses were in the range 4-10 $\mu \mathrm{m}$. The MOVPE growth was carried out at $340{ }^{\circ} \mathrm{C}$ for 3-5 h using dimethylcadmium and diisopropiyl tellurium as precursors. The recrystallization was carried out by scanning the layer surface with a spot of $100 \mu \mathrm{m}$ diameter from an $\mathrm{Ar}$ ion laser beam $(\lambda=514.4 \mathrm{~nm})$. The laser power and time of irradiation were varied in the ranges $200-400 \mathrm{~mW}$ and $30-$ $120 \mathrm{~s}$, respectively. Further experimental details on the laser recrystallization procedure have been reported elsewhere. ${ }^{8}$

CL spectra were recorded in a Hitachi S2500 scanning electron microscope at $80 \mathrm{~K}$ using an ADC Ge detector, from both as-grown and recrystallized epilayers. The details of the experimental setup for CL have been described elsewhere. ${ }^{14}$ As described in this latter work, CL spectra were recorded under different excitation conditions of the electron beam to account for the radiative centers present in a lower concentration. Two different diffraction gratings were used to cover the spectral range from 0.6 to $1.6 \mathrm{eV}$.

Figure 1 shows the effect of the laser recrystallization procedure on the surface microrelief of the $\mathrm{CdTe} / \mathrm{GaAs}$ MOVPE layer. As shown in Fig. 1(a), the as-grown surface has a rough microrelief with features of $0.5-1 \mu \mathrm{m}$ height, which is typical for these layers. ${ }^{6}$ After the recrystallization [Fig. 1(b)], the surface morphology improves uniformly within the entire layer area and the surface roughness decreases by a factor of 10 . The recrystallized surface exhibits submicrometer steps, which are similar to those observed early on the surface of laser recrystallized CdTe/sapphire MOVPE layers. ${ }^{8}$

CL spectra recorded from the as-grown and recrystallized CdTe/GaAs MOVPE layers are shown in Fig. 2. Figure 2 shows the spectra recorded under high excitation density conditions. The as-grown samples exhibit band-edge emission $(1.52 \mathrm{eV})$ along with several broadbands associated to the different deep levels related to the defect structure. These luminescence bands peaked at about 1.4, 0.93, 0.82, 0.77, 

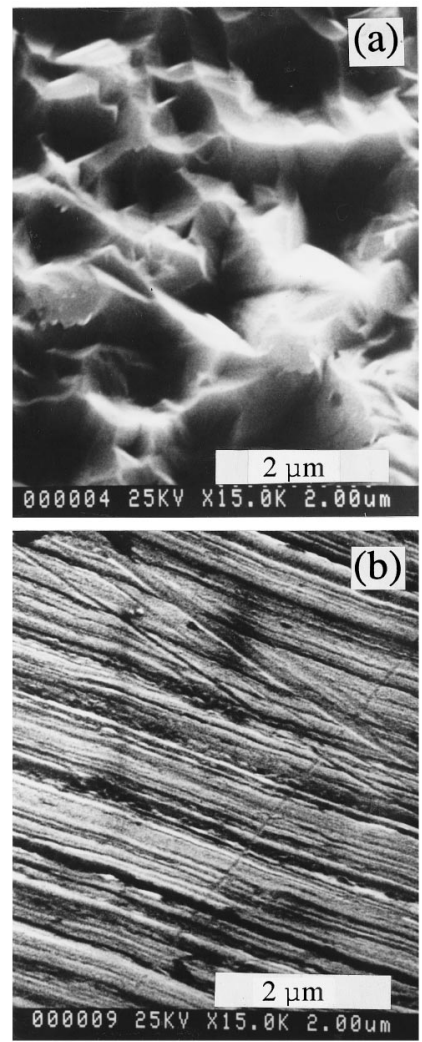

FIG. 1. Surface microrelief of a CdTe MOVPE layer grown on a GaAs substrate: (a) as-grown and (b) after laser recrystallization.

and $0.66 \mathrm{eV}$, have been previously studied in CdTe bulk crystals and attributed to different defect centers. ${ }^{12,15-18}$ The highest relative intensity corresponds to the bands peaked at 1.4 and $0.66 \mathrm{eV}$. After laser recrystallization, a bright region surrounding the irradiation spots are observed in the CL images. The most significant changes occurred in the spectral distribution of cathodoluminescence consist of an increase of the relative intensity of the $1.4 \mathrm{eV}$ band attributed to acceptor levels related to cadmium vacancies $\left(V_{\mathrm{Cd}}\right) .{ }^{18}$ Besides these changes in the intensity of the $1.4 \mathrm{eV}$ band, an increase of the relative intensity of the $1.15 \mathrm{eV}$ band attributed to $V_{\mathrm{Te}}$ (Ref. 18) is also observed. This last effect is more apparent

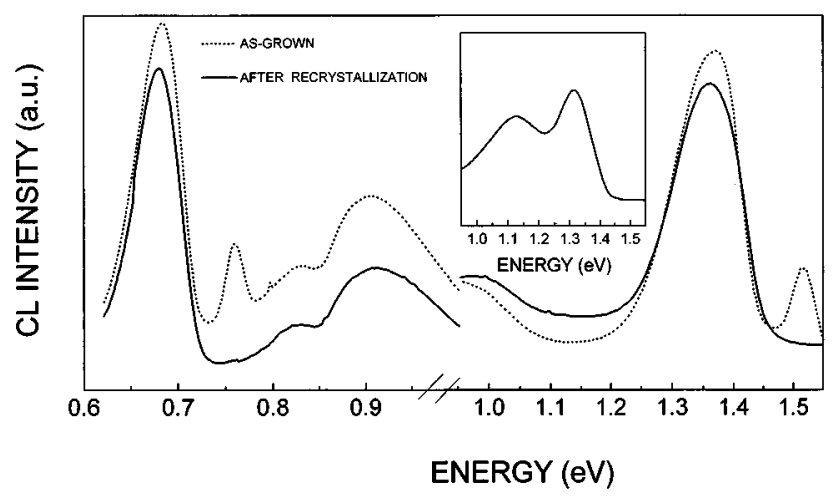

FIG. 2. CL spectra recorded under high excitation conditions of the electron beam from the as-grown and recrystallized CdTe/GaAs MOVPE layers in the spectral range of (a) $0.6-1.0 \mathrm{eV}(1300-2000 \mathrm{~nm})$ and (b) $1.0-1.6 \mathrm{eV}$ $(800-1300 \mathrm{~nm})$. The inset shows the spectrum recorded after laser treatment under low excitation conditions of the electron beam for the high-energy region.

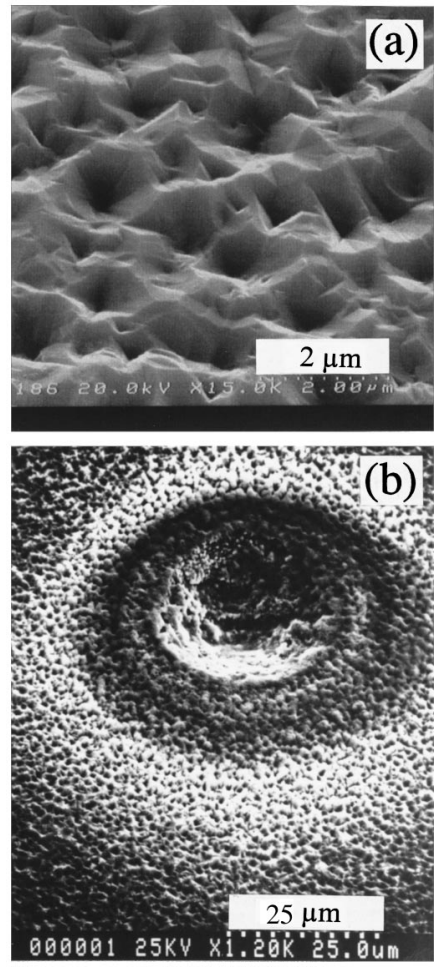

FIG. 3. Surface microrelief of a CdTe MOVPE layer grown on a CdTe substrate: (a) as-grown and (b) after laser recrystallization.

when the spectra are recorded under low excitation density conditions of the electron beam (Fig. 2, inset).

In the low-energy region of the spectrum, an intensity decrease of the bands peaked at $0.66,0.82$, and $0.93 \mathrm{eV}$ is accompanied by the quenching of the $0.77 \mathrm{eV}$ band. A similar quenching effect has already been reported for CdTe bulk crystals submitted to ion-beam irradiation. ${ }^{19}$ The mechanism proposed in that work to explain the changes induced by ion irradiation involves cadmium vacancies as well as extended defects. Cd atoms displaced from their sites by the incident ions would be forced to diffuse searching for a new equilibrium position at a cadmium vacancy. The extended defects present in the material would constitute a favorable hopping path for Cd atoms. As suggested in Ref. 15, a plausible candidate to explain the $0.77 \mathrm{eV}$ emission is a center involving some impurity in the $\mathrm{Cd}$ site. Hence, partial filling of $\mathrm{Cd}$ vacancies would hamper the formation of these centers with the subsequent decrease in the emission. Also, impurity gettering at the interface during the recrystallization process would lead to a lower concentration of impurities in the layer and then to a lower probability of formation of these centers. In our case, CL images recorded from nonirradiated as well as irradiated areas reveal the existence of dark spot features associated with the presence of dislocations, which would serve as preferential diffusion paths. Additionally, diffusion of $\mathrm{Cd}$ atoms would be enhanced by the laser irradiation.

To bring out the substrate effect, CdTe/CdTe MOVPE epitaxial layers were also investigated. Though no lattice mismatch is present, the surface roughness is even higher than that observed for CdTe/GaAs epilayers [Fig. 3(a)]. This rough surface might be caused by thermal strains at the substrate area due to the low thermal conductivity of CdTe, which would magnify any temperature inhomogeneity in the 


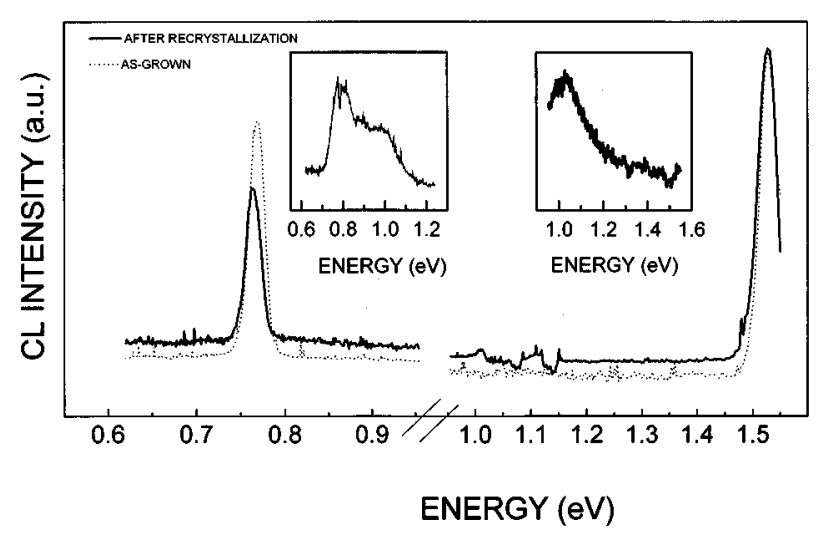

FIG. 4. CL spectra recorded under high excitation conditions of the electron beam from the as-grown and recrystallized CdTe/CdTe MOVPE layers in the spectral range of (a) $0.6-1.0 \mathrm{eV}(1300-2000 \mathrm{~nm})$ and (b) $1.0-1.6 \mathrm{eV}$ (800-1300 nm). In both cases, the insets show the spectra recorded after laser irradiation by decreasing the excitation density of the electron beam.

MOVPE reactor. ${ }^{6}$ The presence of Te precipitates already reported for this material ${ }^{9}$ could also play an important role in the apparition of the mentioned strains. ${ }^{6}$ As shown in Fig. 3 (b), laser irradiation in the same conditions described for $\mathrm{CdTe} / \mathrm{GaAs}$ epilayers causes a strong damage in the $\mathrm{CdTe} /$ CdTe epilayers, while outside the irradiated area the relief remains unchanged. These differences are also evident from the CL spectra. Figure 4 shows the spectra recorded under high excitation density conditions of the electron beam, both before and after laser treatment. Only two peaks, $0.77 \mathrm{eV}$ and band-edge emission, are observable. In nonirradiated areas, no additional bands were revealed by decreasing the excitation density, suggesting a very low intensity of any other emission. However, after irradiation, the different deep-level bands already observed for the $\mathrm{CdTe} / \mathrm{GaAs}$ sample become apparent when low excitation conditions are used (Fig. 4, insets). The irradiation caused a strong increase of the $0.8-1.0 \mathrm{eV}$ emissions, while no $V_{\mathrm{Cd}}$ related peak (1.4 $\mathrm{eV}$ ) is observed. Also, the quenching of the $0.77 \mathrm{eV}$ is not so dramatic as in the layers deposited onto GaAs. These results resemble those obtained for CdTe bulk crystals annealed in cadmium vapor, in which the relative intensity of the $1.4 \mathrm{eV}$ band is very low while the bands in the $0.8-1.0 \mathrm{eV}$ range exhibit the highest intensity. ${ }^{20}$ This similarity between the spectra from irradiated $\mathrm{CdTe} / \mathrm{CdTe}$ layers and $\mathrm{Cd}$ vapor annealed CdTe bulk crystals reinforces our hypothesis about the relation of the $0.77 \mathrm{eV}$ emission with impurity atoms either as substitutional atoms or as complex centers involving cadmium vacancies. The fact that the intensity of the $0.77 \mathrm{eV}$ band does not change drastically, in contrast to the observations made in $\mathrm{CdTe} / \mathrm{GaAs}$ layers, could be explained by the partial filling of $\mathrm{Cd}$ vacancies with $\mathrm{Cd}$ atoms hopping from the substrate, since part of the favorable sites for $\mathrm{Cd}$ diffusion are already occupied, subsequent laser irradiation would not enhance $\mathrm{Cd}$ diffusion significantly and no drastic changes in the $0.77 \mathrm{eV}$ emission should be expected.

In summary, we have shown that under similar laser irradiation conditions, surface morphology and luminescence properties of the epitaxial layers grown on GaAs change dramatically, while the properties of the layers grown on CdTe maintain practically unmodified except in severely damaged regions. The striking differences observed in both kinds of samples are explained by differences in the thermal conductivity of the substrates. Laser induced recrystallization seems to be a good procedure to improve the surface quality of epilayers when the thermal conductivity of the substrate is moderately high (as GaAs in this work). However, when low thermal conductivity substrates (e.g., CdTe) are used, the local damage induced by the laser irradiation is too severe, and no bettering of the surface is achieved.

Cathodoluminescence spectra enable us to study the effect of laser recrystallization as well as the influence of the substrate on the defect structure of CdTe layers. To account for it, mechanisms involving diffusion of cadmium atoms and partial filling of cadmium vacancies are suggested.

One of the authors (N.V.S.) acknowledges the visiting professor fellowship from Universitat de Valencia (Spain). This work has been partially supported by Spanish Project Nos. GV-2205/94 and PB-93-1256.

${ }^{1}$ S. J. Irvine, in Narrow Gap Cadmium-based compounds, edited by P. Capper (INSPEC, Institution of Electrical Engineers, London, 1994), pp. 24-29, and references therein.

${ }^{2}$ D. Rajavel and J. J. Zinck, Appl. Phys. Lett. 63, 322 (1993).

${ }^{3}$ N. V. Sochinskii, S. Bernardi, E. Diéguez, P. Franzosi, and S. V. Kletskii, J. Cryst. Growth 149, 35 (1995).

${ }^{4}$ N. V. Sochinskii, J. C. Soares, M. F. da Silva, P. Franzosi, S. Bernardi, and E. Diéguez, J. Cryst. Growth 161, 195 (1996).

${ }^{5}$ T. Okamoto, T. Saito, S. Murakami, H. Nishino, K. Maruyama, Y. Nishijima, H. Wada, M. Nagashima, and Y. Nogami, Appl. Phys. Lett. 69, 677 (1996).

${ }^{6}$ N. V. Sochinskii, V. Muñoz, V. Bellani, L. Viña, E. Diéguez, E. Alves, M. F. da Silva, J. C. Soares, and S. Bernardi, Appl. Phys. Lett. 70, 1314 (1997).

${ }^{7}$ N. V. Sochinskii, E. Diéguez, S. Bernardi, J. C. Soares, and P. Franzosi, Mater. Sci. Forum 182-184, 167 (1995).

${ }^{8}$ N. V. Sochinskii, E. Diéguez, E. Alves, M. F. da Silva, J. C. Soares, S. Bernardi, J. Garrido, and F. Agulló-Rueda, Semicond. Sci. Technol. 11, 248 (1996).

${ }^{9}$ N. V. Sochinskii, M. D. Serrano, E. Diéguez, F. Agulló-Rueda, U. Pal, J. Piqueras, and P. Fernández, J. Appl. Phys. 77, 2806 (1995).

${ }^{10}$ N. V. Sochinskii, E. Diéguez, U. Pal, J. Piqueras, P. Fernández, and F. Agulló-Rueda, Semicond. Sci. Technol. 10, 870 (1995).

${ }^{11}$ G. Panin, J. Piqueras, N. V. Sochinskii, and E. Diéguez, Mater. Sci. Eng. B 42, 277 (1996).

${ }^{12}$ U. Pal, P. Fernández, J. Piqueras, N. V. Sochinskii, and E. Diéguez, J. Appl. Phys. 78, 1992 (1995).

${ }^{13}$ G. Panin, J. Piqueras, N. V. Sochinskii, and E. Diéguez, Appl. Phys. Lett. 70, 877 (1997).

${ }^{14}$ F. Domínguez-Adame, J. Piqueras, and P. Fernández, Appl. Phys. Lett. 58, 257 (1991).

${ }^{15}$ T. Takebe, J. Saraie, and H. Matsunami, J. Appl. Phys. 53, 457 (1982).

${ }^{16}$ A. Castaldini, A. Cavallini, B. Fraboni, L. Polenta, P. Fernández, and J. Piqueras, Appl. Phys. Lett. 69, 3510 (1996).

${ }^{17}$ A. Castaldini, A. Cavallini, B. Fraboni, L. Polenta, P. Fernández, and J. Piqueras, Mater. Sci. Eng. B 42, 302 (1996).

${ }^{18}$ W. Stadler, d. M. Hoffman, H. C. Alt, T. Muschik, B. K. Meyer, E. Weigel, G. Müller-Vogt, M. Salk, E. Rupp, and K. W. Benz, Phys. Rev. B 51, 10619 (1995).

${ }^{19}$ G. Panin, P. Fernández, and J. Piqueras, Semicond. Sci. Technol. 11, 1354 (1996).

${ }^{20}$ U. Pal (unpublished work). 